Palavras chave:

Khaya ivorensis

Khaya senegalensis

Propriedades da madeira

Histórico:

Recebido 03/09/2014

Aceito 15/1 I/2015

Keywords:

Khaya ivorensis

Khaya senegalensis

Wood Properties

Correspondência: tamarasfamorim@gmail.com
Tâmara Suely Filgueira Amorim França', Marina Donária Chaves Arantes², Juarez Benigno Paes $^{2}$, Graziela Baptista Vidaurre², José Tarcísio da Silva Oliveira² ${ }^{2}$ Edy Eime Pereira Baraúna ${ }^{3}$

\title{
CARACTERÍSTICAS ANATÔMICAS E PROPRIEDADES FÍSICO-MECÂNICAS DAS MADEIRAS DE DUAS ESPÉCIES DE MOGNO AFRICANO
}

RESUMO: O objetivo do trabalho foi avaliar as características anatômicas e propriedades físico-mecânicas das madeiras de duas espécies de mogno africano (Khaya ivorensis e Khaya senegalensis). Foram utilizadas cinco árvores de cada espécie com 19 anos de idade, proveniente de parcelas experimentais implantadas nas áreas da Reserva Natural Vale, Sooretama, Espírito Santo. Para a característica anatômica foram utilizadas amostras retiradas na posição de transição entre cerne e alburno (cerne periférico). Na avaliação da massa específica básica foram empregadas duas cunhas opostas dos discos obtidos na região correspondente ao diâmetro a altura do peito (DAP), tomado a I,30 m do solo, e nas posições $0,25,50,75$ e $100 \%$ da altura comercial da árvore. Para a retratibilidade foram retiradas 20 amostras obtidas aleatoriamente ao longo do tronco de cada espécie. Os ensaios mecânicos foram realizados em amostras obtidas do pranchão central, ao ser descartada a medula. As espécies foram diferentes quanto ao tipo e frequência de vasos e porosidade. Os raios foram predominantemente multisseriados e heterocelulares e as fibras libriformes. A madeira de Khaya ivorensis possui massa específica baixa, enquanto a de Khaya senegalensis, massa específica média. As duas espécies possuem estabilidade dimensional normal. Em relação as propriedades mecânicas, a madeira de Khaya senegalensis teve os maiores valores para todos os ensaios realizados.

\section{ANATOMICAL CHARACTERISTICS AND WOOD PHYSICAL- MECHANICAL PROPERTIES OF TWO AFRICAN MAHOGANY SPECIES}

ABSTRACT: The objective of this study was to evaluate the anatomical characteristics and wood physical-mechanical properties of two African mahogany species (Khaya ivorensis and Khaya senegalensis). Five trees of each species with 19-years-old, proceeding from experimental parcels in the areas of the Reserva Natural Vale, located in Sooretama, Espírito Santo State, Brazil. For the anatomical characteristics samples were taken at the position of transition between heartwood and sapwood (peripheral heart). In the evaluation of the specific density were used two opposing wedges from discs gotten in the corresponding region to the diameter the height of the breast (DHB), taken the I,30 $\mathrm{m}$ of the ground, and in positions $0,25,50,75$ and $100 \%$ of the commercial height of the tree. For shrinkage 20 samples obtained randomly throughout the trunk of each species. The mechanical tests were carried through in removed samples of the central large plank, to the discarded the pith. The species were different how much to the type and frequency of vases and porosity. Rays were predominantly multiseriates, heterocellulars and fibers are libriform. The wood of Khaya ivorensis has low specific density and wood of Khaya senegalensis has medium specific density. The two wood species have normal dimensional stability. In relation the mechanical properties, the Khaya senegalensis wood had the biggest values for all the carried through tests.

\footnotetext{
' Mississippi State University - Starkville, Estados Unidos

2 Universidade Federal do Espírito Santo - Jerônimo Monteiro, Espirito Santo, Brasil

${ }^{3}$ Universidade Federal de Minas Gerais - Montes Claros, Minas Gerais, Brasil
} 


\section{INTRODUÇÃO}

O gênero Khaya pertence à família Meliaceae e de acordo com Barroso ( 1984 ) compreende 5 I gêneros que abrangem I .400 espécies, em que $60 \%$ delas fazem parte da economia florestal de vários países. Pinheiro et al. (20II) afirmaram que o gênero Khaya possui quatro importantes espécies produtoras de madeira comerciais na África, Comores e Madagascar, sendo conhecidas como mogno africano sendo semelhantes aos mognos latino-americano (Swietenia macrophylla, Swietenia mahogani e Swietenia humilis).

No Brasil, a introdução e o cultivo de meliáceas exóticas em substituição principalmente ao mogno nativo (Swietenia macrophylla) são difundidos e recomendados principalmente em função da grande semelhança entre as espécies, proibição da exploração e comercialização do mogno nativo e resistência ao ataque da Hypsipyla grandella, conhecida como broca das meliáceas ou broca do ponteiro (CASTRO et al., 2008), por atacar os brotos terminais da planta impedindo seu desenvolvimento e, tornado impossível a produção florestal, principalmente em plantios puros.

As espécies Khaya ivorensis e Khaya senegalensis, vulgarmente conhecidas como mogno africano, são arbóreas de grande porte e produtoras de madeira de boa qualidade. São de alto valor comercial e sua madeira é utilizada para construção naval, fabricação de móveis, pisos, decoração de ambientes internos e externos (ARNOLD, 2004). No Brasil os plantios de mogno africano estão distribuídos pelos estados do Espírito Santo, São Paulo, Minas Gerais, Mato Grosso do Sul, Bahia e Paraná (CARVALHO, 20I I).

Para a correta utilização da madeira, é de extrema necessidade o conhecimento das características anatômicas, físicas e mecânicas do material, pois estas informações possibilitam a determinação das propriedades tecnológicas, a fim de utilizar o material com economicidade e segurança (OLIVEIRA, 2007).

Dias et al. (2012) constataram que a madeira de mogno africano possui grande potencial econômico, no entanto há carência de conhecimento tecnológico e científico da madeira. Desta forma, o objetivo deste trabalho foi avaliar as propriedades anatômicas e físicomecânicas das madeiras de duas espécies de mogno africano (Khaya ivorensis e Khaya senegalensis).

\section{MATERIAL E MÉTODOS}

O material estudado foi procedente das espécies Khaya ivorensis A. Chev. e Khaya senegalensis (Desv.) A. Juss., com idade de 19 anos, proveniente de parcelas experimentais da Reserva Natural Vale, Sooretama, norte do Estado do Espírito Santo.

Foram coletadas cinco árvores por espécie e retirados discos na região correspondente ao diâmetro à altura do peito (DAP), tomado a $1,30 \mathrm{~m}$ do solo e nas posições 0, 25, 50, 75 e $100 \%$ da altura comercial, considerada a partir da base até o local de inserção do primeiro galho em cada tronco.

Após a retirada dos discos, foram obtidas cinco toras com comprimentos e diâmetros variáveis. Cada tora foi desdobrada e transformada em tábuas com espessura e largura variáveis, além de um pranchão central.

\section{Descrição anatômica das madeiras estudadas}

Foram retirados corpos de prova dos discos do DAP com de 2,0 × 2,0 x 2,0 cm, nos planos transversal, longitudinal tangencial e radial. Na descrição microscópica seguiram-se os procedimentos da norma do International Association of Wood Anatomists Committee - IAWA ( I 989). A determinação da cor da madeira seca foi realizada de acordo com a escala de Munsell (1957).

Para a obtenção das seções histológicas, os corpos de prova foram amolecidos em água à temperatura de ebulição. As secções foram retiradas em micrótomo de deslize, com espessura variando de I 8 a $20 \mu \mathrm{m}$.

Os cortes transversal, longitudinal tangencial e radial, foram submetidos ao processo de coloração com safranina aquosa a $1 \%$ e, posteriormente preparadas lâminas permanentes. A dissociação dos elementos celulares foi realizada de acordo com o método Franklin (1945), tendo o material dissociado sido corado com safranina a 1\%. As lâminas semi-permanentes contendo o material dissociado foram preparadas em solução aquosa de glicerina ( $I: I, v: v)$. Foram estudados os elementos de vasos, parênquima axial, raios e fibras.

\section{Propriedades físicas e mecânicas da madeira}

Para determinar a massa específica básica foram utilizadas duas cunhas opostas obtidas do disco retirado no DAP e nas posições 0, 25, 50, 75 e 100\% da altura comercial de cinco árvores de cada espécie. $\bigcirc$ 
ensaio foi realizado de acordo com a Norma Brasileira Regulamentadora - NBR II94I, da Associação Brasileira de Normas Técnicas - ABNT (2003).

Para obtenção da retratibilidade das madeiras foram utilizadas 20 amostras com dimensões nominais de 2,0 × 2,0 × 3,0 cm (radial $\times$ tangencial $\times$ longitudinal) para cada espécie, retiradas aleatoriamente ao longo do tronco. O ensaio foi realizado de acordo com a NBR 6230 da ABNT ( 1 982).

As propriedades mecânicas determinadas foram o módulo de elasticidade (MOE) e de ruptura (MOR) à flexão estática, resistência à compressão axial, ao cisalhamento e dureza Janka. Estes ensaios foram realizados de acordo com a American Society for Testing and Materials - ASTM D - I43 (2008), em amostras retiradas do pranchão central. Decidiu-se empregar a norma americana, pelas características da madeira e dimensões das amostras, bem como, em decorrências do uso da mesma em outros trabalhos em que foram utilizadas madeiras de mogno africano.

Os ensaios de resistência mecânica foram realizados em máquina universal de ensaio com capacidade de 10 toneladas, com sistema de aquisição de dados automatizado. Após a realização dos ensaios, as amostras foram pesadas para a determinação da umidade e os valores ajustados ao padrão de $12 \%$, de acordo com a NBR 7I90 da ABNT (1997).

\section{Análise estatística}

Para comparar as características anatômicas quantitativas, a estabilidade dimensional (retratibilidade e fator de anisotropia) e as propriedades mecânicas das duas espécies estudadas foi empregado um delineamento inteiramente casualizado.

Realizaram-se os testes de Cochran e Bartlett (homogeneidade de variância) e de Lilliefors (normalidade) e empregou-se a análise de variância e, quando detectada diferença significativa entre as espécies para as características avaliadas pelo teste $F$ ( $p \geq 0,05$ ), as médias foram comparadas, uma vez que foram empregados apenas dois tratamento (espécies).

\section{RESULTADOS E DISCUSSÃO}

\section{Características anatômicas das espécies estudadas}

A madeira de Khaya ivoresnsis possui coloração rosada, 7,5YR 8/3, de acordo com a escala de Munsell (1957). Possui brilho moderado, cheiro imperceptível, gosto ligeiramente amargo e as camadas de crescimento são pouco distintas.

Os vasos são de seção ovalada, predominantemente solitários, com presença de múltiplos de dois e três. São obstruídos por gomo resina, com porosidade é em anel semicircular. Quanto à freqüência, os vasos são pouco numerosos $\left(5,9\right.$ poros $\left.\cdot \mathrm{mm}^{-2}\right)$, com diâmetro tangencial médio ( I 32,3 $\mu \mathrm{m})$ (Figura I).

O parênquima é apotraqueal difuso escasso. Os raios são multisseriados, heterogêneos, formados por células eretas, quadradas e procumbentes, possuindo estrutura não estratificada, sendo pouco numerosos $\left(5,6\right.$ raios $\left.\mathrm{mm}^{-2}\right)$, finos e muito altos, com
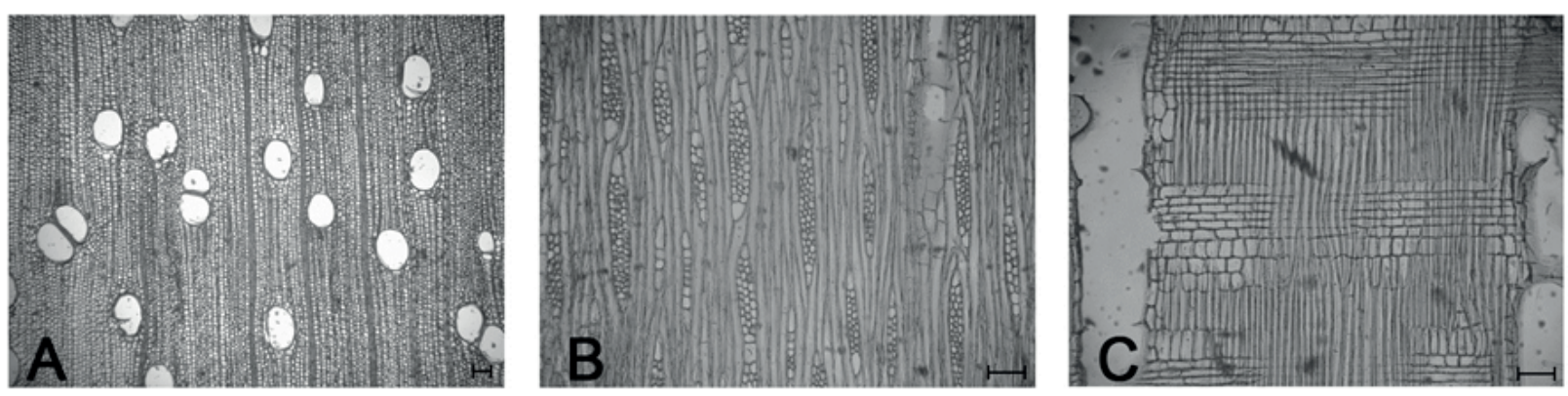

Figura I Fotomicrografia ilustrando os três planos de observação da madeira de Khaya ivorensis: a) plano transversal; b) plano longitudinal tangencial; e c) plano longitudinal radial. Barra $=100 \mu \mathrm{m}$.

Figure I Photo micrograph illustrating the three observation plans of the Khaya ivorensis wood: a) transversal plan; b) tangential longitudinal plan; and c) plain longitudinal radial. Bar $=100 \mu \mathrm{m}$. 
altura de 486,9 $\mu \mathrm{m}$ e número de células de 16, I; largura de $54,0 \mu \mathrm{m}$ e número de células de 3,2. As fibras são libriforme e curtas ( I,2 $\mu \mathrm{m})$, largura de 27, I $\mu \mathrm{m}$, com 18,9 $\mu \mathrm{m}$ de diâmetro de lume e $4,4 \mu \mathrm{m}$ de espessura de parede.

A madeira de Khaya senegalensis possui cerne e alburno pouco distinto. A madeira de cerne tem coloração vermelho claro, 2,5YR 6/6 de acordo com a escala de Munsell (1957). Possui brilho moderado, cheiro imperceptível, gosto ligeiramente amargo e com camadas de crescimento pouco distintas. A porosidade é difusa, com os vasos obstruídos por goma, sendo múltiplos de dois e três (Figura 2).
Quanto à frequência, são pouco numerosos (8,8 poros $\left.\mathrm{mm}^{-2}\right)$, com diâmetro tangencial pequeno $(98,7 \mu \mathrm{m})$. O parênquima é paratraqueal do tipo vasicêntrico e aliforme escasso, ocorrendo também parênquima apotraqueal marginal.

Os raios são multisseriados, heterogêneos, formados por células eretas, quadradas e procumbentes, possuindo estrutura não estratificada e pouco numerosos $\left(7,2\right.$ raios $\left.\mathrm{mm}^{-2}\right)$, estreitos e muito altos, com altura de 34I,3 $\mu \mathrm{m}$ e em número de células de II,9; com largura de $70 \mu \mathrm{m}$ e em número de células de 3,6. As fibras são libriformes, curtas (I,2 $\mu \mathrm{m})$, largura de $20,5 \mu \mathrm{m}$, com diâmetro de lume de I 0,6 $\mu \mathrm{m}$ e espessura de parede de $4,9 \mu \mathrm{m}$.
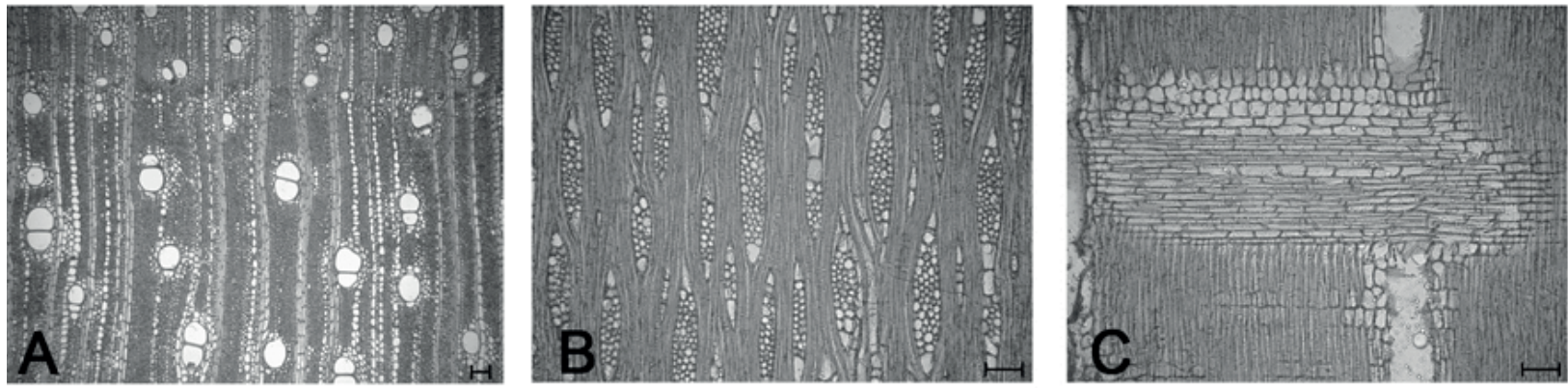

Figura 2 Fotomicrografia ilustrando os três planos de observação da madeira de Khaya senegalensis: a) plano transversal; b) plano longitudinal tangencial; e c) plano longitudinal radial. Barra $=100 \mu \mathrm{m}$.

Figure 2 Photo micrograph illustrating the three observation plans of the Khaya senegalensis wood: a) transversal plan; b) tangential longitudinal plan; and c) plain longitudinal radial. Bar $=100 \mu \mathrm{m}$.

A madeira de Khaya ivorensis proveniente da África Ocidental possui vasos de diâmetro médio de $100 \mu \mathrm{m}$, predominantemente solitários e alguns múltiplos de dois e três; raios heterogêneos, com cinco células de largura, 18 de altura e fibras septadas, possuindo comprimento médio de I,4 mm (AYENSU; BENTUM, 1974). A porosidade é difusa, diâmetro de vaso de $192 \mu \mathrm{m}$, algumas vezes obstruídos com gomo resina, parênquima paratraqueal vasicêntrico e raios multisseriados heterogêneos, com 5 a 8 células de largura (RINNE et al., 20I I).

A madeira de Khaya senegalensis possui porosidade difusa, vasos múltiplos, frequência de 6,5 vasos $\mathrm{mm}^{-2}$; diâmetro médio de $170 \mu \mathrm{m}$, com presença de gomo resina nos mesmos; parênquima paratraqueal vasicêntrico e apotraqueal concêntrico; raios multisseriados, heterogêneos, com 8 , I células de largura, e fibras septadas, com parede espessa (DONKOR, 1997).

Notou-se que as duas espécies estudadas possuem camadas de crescimento distintas, diferença entre cerne e alburno, com brilho moderado, cheiro imperceptível e gosto ligeiramente amargo e a cor varia entre rosa ao avermelhado.

Observou-se que a coloração do cerne é variável entre as espécies estudadas; as camadas de crescimento em ambas as madeiras são pouco distintas; elas possuem tipos diferentes de parênquima axial; o arranjo, frequência, diâmetro dos poros. No entanto, os raios semelhantes, sendo basicamente multisseriados e heterogêneos, possuindo células com formatos quadrado, ereto e procumbente e as fibras de ambos os lenhos, são do tipo libriforme.

\section{Propriedades físicas das madeiras estudadas}

Houve diferença estatística entre a massa específica das madeiras de ambas as espécies de mogno africano (Tabela I). De acordo com a classificação proposta por Marques et al. (1997), a madeira de Khaya ivorensis possui massa específica baixa e a de 
Tabela I Valores médios de massa específica básica e retratibilidade das madeiras de Khaya ivorensis e Khaya senegalensis.

Table I Mean values of specific gravity and shrinkage of Khaya ivorensis and Khaya senegalensis woods.

\begin{tabular}{cccccccc}
\hline \multirow{2}{*}{ Espécie } & $\begin{array}{c}\text { Massa } \\
\text { Específica Básica } \\
\left(\mathrm{g} \cdot \mathrm{cm}^{-3}\right)\end{array}$ & Radial & Tangencial & Longitudinal & Volumétrica & T/R \\
\cline { 3 - 7 } K. ivorensis & $0,49 \mathrm{Ib}$ & $3,39 \mathrm{a}$ & $5,58 \mathrm{a}$ & $0,2 \mathrm{la}$ & $9,18 \mathrm{a}$ & $\mathrm{I}, 65 \mathrm{~b}$ \\
K. senegalensis & $0,588 \mathrm{a}$ & $3, \mathrm{Il} \mathrm{b}$ & $5,57 \mathrm{a}$ & $0,30 \mathrm{a}$ & $8,98 \mathrm{~b}$ & $\mathrm{I}, 79 \mathrm{a}$ \\
\hline
\end{tabular}

Médias seguidas pela mesma letra não diferem entre si (Teste F; $\mathrm{p}>0,05)$.

Khaya senegalensis, massa específica média.

Para a madeira de Khaya ivorensis nativas de florestas naturais da República dos Camarões e de Angola foi obtida massa específica básica de $0,48 \mathrm{~g} \cdot \mathrm{cm}^{-3}$ (LEMMENS, 2008). Para Khaya ivorensis, com 10 anos de idade, plantada em Seropédica, RJ, encontraram massa específica básica de $0,47 \mathrm{~g} \cdot \mathrm{cm}^{-3}$ (CARVALHO et al., 2010).

Para a madeira de Khaya senegalensis com 18 anos de idade, provenientes de Queensland, Austrália, foram encontraram valores de massa específica básica de $0,67 \mathrm{~g} \cdot \mathrm{cm}^{-3}$ (ZBONAK et al., 2004). Para a qualidade da madeira de 38 árvores de Khaya senegalensis, com 36 anos, provenientes de Brisbane, Austrália, foram encontrados valores de massa específica básica de $0,63 \mathrm{~g} \mathrm{~cm}-3$ (ARMSTRONG et al., 2004). Ambos os valores são superiores ao deste estudo, provavelmente em consequência da qualidade do sítio e da idade das plantas estudadas.

A madeira de Khaya senegalensis estudada, apesar de possuir maior frequência de vasos e de raios e, maior largura dos mesmos quando comparada com a Khaya ivorensis, a mesma apresentou vasos de menores diâmetros, raios de menor altura, largura e com menor número de células por camada, com fibras de menor largura, menor diâmetro de lume e consequentemente, maior espessura de parede, o que lhe conferiu uma maior massa específica e menor estabilidade dimensional a sua madeira.

Os valores de massa específica básica evidenciaram variações no sentido longitudinal do tronco, indicadas pelas diferentes porcentagens da altura comercial das árvores estudadas (Figura 3).

As espécies possuem comportamento semelhante, sendo a massa específica básica decrescente da base para a posição de $50 \%$ da altura comercial e, posteriormente, crescente deste ponto até 100\% da altura da altura comercial. Mesmo as duas espécies possuindo a mesma tendência de variação da massa específica no sentido base topo, a madeira de Khaya ivorensis possui maior incremento da massa específica do DAP até a posição de $100 \%$, sendo o aumento desta madeira de $8 \%$, enquanto a da Khaya senegalensis foi de $3 \%$.

A madeira de Khaya ivorensis possui madeira com menor massa específica, sendo mais indicada para usos que não requerem alta resistência mecânica, como produção de móveis, compensados e acabamento superficial em construção civil. Para madeira de Khaya senegalensis observou-se que a mesma produziu madeira de maior massa específica, portanto, mais adequada para usos que requerem maior resistência mecânica das peças.

Em relação à retratibilidade, houve diferença significativa entre as espécies na retração radial, volumétrica e no fator anisotrópico. A madeira de Khaya ivorensis obteve maiores valores médios nas retrações radial, tangencial e fator anisotrópico. Em relação à anisotropia, mesmo havendo diferença significativa entre as espécies, de acordo com a classificação de Durlo e Marchiori (1992) e Logsdon e Penna (2005), ambas as espécies possuem média estabilidade dimensional.

Os valores médios de retração radial, tangencial e fator anisotrópico de 3,7; 5,5; e I,5, respectivamente para a madeira de Khaya ivorensis, citados pelo Forest Product Laboratory - FPL (2010a) e valores médios de retração radial (4,5\%), tangencial $(2,5 \%)$ e fator anisotrópico $(1,8)$ da madeira de Khaya senegalensis, divulgados pelo Centre de Cooperation Internationale en

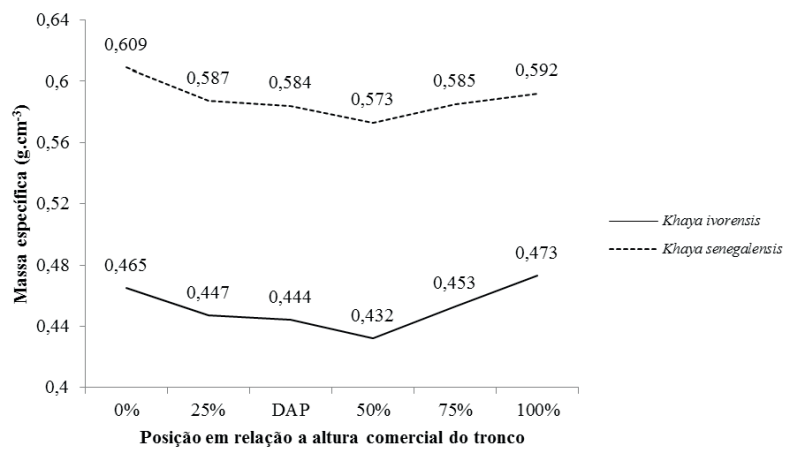

Figura 3 Variação da massa específica básica ao longo do fuste das madeiras de Khaya ivorensis e Khaya senegalensis.

Figure 3 Variation of specific density along the stem to Khaya ivorensis and Khaya senegalensis woods. 
Recherche Agronomique pour le Développement - CIRAD (2012) foram semelhantes aos valores encontrados neste estudo. No entanto, os valores de retração volumétrica de $16,8 \%$ para a madeira de Khaya ivorensis, com 10 anos proveniente de uma parcela experimental em Seropédica, RJ (CARVALHO et al., 20I0), foram superiores aos obtidos nesta pesquisa. Isso ocorreu provavelmente, por causa da maior proporção de lenho adulto na madeira das árvores estudadas (19 anos).

Os valores de retração radial, tangencial e longitudinal de I,8; 2,5; e 0,3\%, respectivamente (ARMSTRONG et al., 2004) para a madeira de Khaya senegalensis (38 anos), proveniente de plantio no Território Norte, Austrália, foram inferiores aos valores encontrados neste estudo. Estes resultados podem ser explicados em função da qualidade de sítio, características edafoclimáticas e idade da madeira, pois a quantidade de lenho adulto confere maior estabilidade dimensional à madeira.

\section{Propriedades mecânicas das madeiras estudadas}

Não houve diferença estatística para o módulo de elasticidade e módulo de ruptura à flexão estática das espécies (Tabela 2). Observa-se que o lenho dessa espécie possui maior resistência mecânica quando comparado ao de Khaya ivorensis, essa superioridade é decorrente da maior espessura da parede de suas fibras, que gerou por consequência, uma maior massa específica e resistência da madeira. Entre as propriedades mecânicas, a maior diferença entre as espécies foi na dureza Janka tangencial, sendo a Khaya senegalensis $47,83 \%$ mais resistente que a Khaya ivorensis.

Segundo a classificação do O Instituto Brasileiro do Meio Ambiente e dos Recursos Naturais Renováveis - IBAMA (2005), madeira de Khaya ivorensis possui alto módulo de ruptura, módulo de elasticidade médio, resistência à compressão axial média, resistência ao cisalhamento alta e dureza paralela às fibras média. A madeira de Khaya senegalensis possui alto módulo de ruptura, módulo de elasticidade médio, resistência à compressão axial média, resistência ao cisalhamento alta e alta resistência à dureza paralela às fibras.

As duas espécies de mogno africano podem ser enquadradas nas mesmas classes para MOR, MOE, compressão axial e cisalhamento, no entanto, existe uma variação na resistência à dureza paralela às fibras. No estudo de critérios para indicar os usos mais adequados para madeiras tropicais, Grobério e Lahr (2002) apontaram a dureza como um parâmetro de grande importância para indicar o emprego de determinadas espécies para pisos. A madeira de Khaya senegalensis, de maior dureza, pode ser indicada para produção de pisos.

Nascimento et al. (1997) agruparam 87 espécies madeireiras em cinco grupos por meio de análise multivariada, em decorrência da massa específica básica e de cinco propriedades mecânicas; módulo de elasticidade, módulo de ruptura, compressão axial, compressão perpendicular às fibras e cisalhamento. De acordo com o agrupamento, a madeira de Khaya ivorensis foi indicada para construção leve e molduras, acabamentos e divisórias, móveis de utilidade e compensado. Enquanto a de Khaya senegalensis para produção de assoalho, construção leve e embarcações.

Os valores médios de resistência mecânica da madeira de Khaya ivorensis e Khaya senegalensis obtidos estão de acordo com os valores encontrados na literatura para exemplares provenientes de florestas nativas da África (FPL, 20I0ab), no entanto, a madeira de Khaya ivorensis possui resistência a dureza superior aos valores encontrados pelo FPL (2010a) e pelo The Wood Data Base (20I2).

De acordo com os dados do Instituto de Pesquisa Tecnológica do Estado de São Paulo - IPT (1989) os valores médios encontrados para Swietenia macrophylla foram módulo de ruptura de 90,6 MPa, módulo de elasticidade de $9.110 \mathrm{MPa}$, compressão axial de 10,9 MPa, cisalhamento de 10,9 MPa, e dureza Janka de 49,4 MPa. Comparando estes dados com

Tabela 2 Propriedades mecânicas das madeiras de Khaya ivorensis e Khaya senegalensis a 12\% de umidade.

Table 2 Mechanical properties of Khaya ivorensis and Khaya senegalensis woods at I2\% of moisture content.

\begin{tabular}{|c|c|c|c|c|c|c|}
\hline \multirow{3}{*}{ Espécie } & \multicolumn{6}{|c|}{ Propriedades Mecânicas (MPa) } \\
\hline & \multicolumn{2}{|c|}{ Flexão Estática } & \multirow{2}{*}{ Compressão } & \multirow{2}{*}{ Cisalhamento } & \multicolumn{2}{|c|}{ Dureza Janka } \\
\hline & MOR & MOE & & & Tangencial & Radial \\
\hline K. ivorensis & $78,4 a$ & $9.577 \mathrm{a}$ & $43, I b$ & $12,6 b$ & $32,4 b$ & $36,8 b$ \\
\hline K. senegalensis & $83,2 a$ & $10.169 a$ & $46,1 \mathrm{a}$ & $18,6 a$ & $62, \mathrm{la}$ & $59,4 a$ \\
\hline
\end{tabular}

Médias seguidas pela mesma letra não diferem entre si (Teste F; $p>0,05$ ). MOR e MOE = Módulos de ruptura e de elasticidade, respectivamente. 
os valores encontrados para as madeiras de mogno africano, a Khaya ivorensis obteve menor valor médio de resistência à dureza Janka e o modulo de ruptura das duas espécies foi inferior ao encontrado para a madeira de Swietenia macrophylla.

\section{CONCLUSÕES}

As duas espécies apresentaram propriedades organolépticas semelhantes. No entanto, a descrição anatômica qualitativa e quantitativa demonstrou haver diferença entre as espécies analisadas. A madeira de Khaya ivorensis possui massa específica básica baixa e a madeira de Khaya senegalensis massa específica básica média. A estabilidade dimensional foi mediana para as duas espécies, com baixos valores de contração volumétrica. A madeira de Khaya senegalensis possui maiores valores para as propriedades mecânicas avaliadas quando comparada à madeira de Khaya ivorensis.

\section{REFERÊNCIAS}

\section{AMERICAN SOCIETY FOR TESTING AND MATERIALS.} ASTM D - 143: standard methods of testing small clear specimens timber. Philadelphia, 2005. 3 I p.

ARMSTRONG, M.; LELIEVRE, T.; REILLY, D.; ROBERTSON, $B$. Evaluation of the wood quality and utilization potential of plantation grown Khaya senegalensis (African mahogany). Mareeba: Department of Primary Industries and Fisheries, 2004. 93p. (RIRDC Project DNT32A - Information Booklet, IB6).

ARNOLD, R. Khaya senegalensis - current use from its natural range and its potential in Sri Lanka and elsewhere in Asia. In: Prospects for high-value hardwood timber plantations in the 'dry' tropics of Northern Australia. Proceedings of a Workshop held in Mareeba. Mareeba: Department of Primary Industries and Fisheries, 2004, p. I-8.

ASSOCIAÇÃO BRASILEIRA DE NORMAS TÉCNICAS ABNT. NBR I I94I: determinação da densidade básica em madeira. Rio de Janeiro, 2003. 6p.

ASSOCIAÇÃO BRASILEIRA DE NORMAS TÉCNICAS - ABNT. NBR 7 190: projetos de estruturas de madeira. Anexo B - Ensaios de caracterização. Rio de Janeiro: 1997. 107p.

ASSOCIAÇÃO BRASILEIRA DE NORMAS TÉCNICAS - ABNT. NBR 6230: ensaios físicos e mecânicos de madeiras. Rio de Janeiro, $1982.89 \mathrm{p}$.

AYENSU, E S.; BENTUM, A. Commercial timbers of West Africa. Washington: Smithsonian Institution Press, 1974. 69p. (Smithsonian Contributions to Botany, 14).

BARROSO, G.M. Sistemática de angiospermas do Brasil. Viçosa: UFV, 1984. v. 2. 377p.
CARVALHO, A. M.; SILVA, B. T. B.; LATORRACA, J. V. F. Avaliação da usinagem e caracterização das propriedades físicas da madeira de mogno africano (Khaya ivorensis A. Chev.). Cerne, v. 16, Suplemento, p. I06-II4, 2010.

CASTRO, C. R. T.; LOURENÇO JÚNIOR, J. B.; SANTOS, N. F. A.; MONTEIRO, E. M. M.; AVIZ, M. A. B.; GARCIA, A. R. Sistema silvipastoril na Amazônia: ferramenta para elevar o desempenho produtivo de búfalos. Ciência Rural, v. 38, n. 8, p. 2395-2402, 2008.

CENTRE DE COOPERATION INTERNATIONALE EN RECHERCHE AGRONOMIQUE POUR LE DÉVELOPPEMENT-DÉPARTEMENT FORÊT - CIRAD. Acajou D'Afrique. Les principales caractéristiques technologiques de 245 essences forestières tropicales. France, 2012. Disponível em <http://tropix.cirad.fr/africa/ ACAJOU_D_AFRIQUE.pdf>. Acesso em: 24 abr. 2013.

DIAS, A. H. S.; UMETSU, F.; BREIER, T. B. Avaliação do potencial de germinação do mogno-africano sob diferentes tipos de substrato e períodos de armazenamento. Informativo ABRATES, v. 22, n. I, p. 26-29, 2012.

DONKOR, B. N. Stem wood structure of four Ghanaian Khaya species. 1997. 97f. Dissertation. Faculty of Forestry, Ottawa, 1997.

DURLO, M. A.; MARCHIORI, J. N. C. Tecnologia da madeira: retratibilidade. Santa Maria: CEPEF/FATEC, 1992. 33p. (Série Técnica, 10).

FOREST PRODUCT LABORATORY - USDA FOREST SERVICE. Wood technology transfer fact sheet Khaya ivorensis. Tropical timbers of the world. Research and Development: Forest Products Laboratory, Madison. 2010a. Disponível em: <http://www.fpl.fs.fed.us/ documnts/TechSheets/Chudnoff/African/htmIDocs_africa/ khayaivor.html>. Acesso em: 24 abr. 2013.

FOREST PRODUCT LABORATORY - USDA FOREST SERVICE. Wood technology transfer fact sheet - Khaya senegalensis. Tropical timbers of the world. Research and Development: Forest Products Laboratory, Madison. 20 IOb. Disponível em: <http://www.fpl.fs.fed.us/documnts/TechSheets/Chudnoff/ African/htmlDocs_africa/khayagrandi.html>. Acesso em: 24 abr. 2013.

FRANKLIN, G. L. Preparation of thin sections of synthetic resins and wood - resin composites, and a new macerating method for wood. Nature, v. I55, n. 3924, p. 5I-5I, 1945.

GROBÉRIO, M. P.; LAHR, F. A. R. Indicações para o emprego da madeira de espécies tropicais do Brasil. Madeira: Arquitetura e Engenharia, v.3, n. 8, p. I-I I, 2002.

INSTITUTO DE PESQUISAS TECNOLÓGICAS DO ESTADO DE SÃO PAULO - IPT. Fichas de características das madeiras brasileiras. 2. ed. São Paulo: IPT, 1989. 4I8p. (Publicação IPT, I79I). 
INSTITUTO BRASILEIRO DE MEIO AMBIENTE E DOS RECURSOS NATURAIS RENOVÁVEIS - IBAMA. Banco de dados de madeiras brasileiras. 2005. Disponível em: <http://www.ibama.gov.br/lpf/madeira/resultado. php?idioma $=$ Português $>$. Acesso em: 28 out. 2013.

INTERNATIONAL ASSOCIATION OF WOOD ANATOMISTS - IAWA. List of microscopic features for hardwood identification. IAWA Bulletin, Leiden, v.I0, n.3, p.226332, 1989

LEMMENS, R. H. M. J. Khaya ivorensis A.Chev. In: LOUPPE, D.; OTENG-AMOAKO, A. A.; BRINK, M. (Eds.). Prota 7 (I): Timbers/Bois d'œuvre I. Wageningen: PROTA, 2008. (CD-Rom).

LOGSDON, N. B.; PENNA, J. E. Análise comparativa entre os coeficientes de anisotropia dimensional da madeira, no inchamento e na retração. Agricultura Tropical, v.8, n. I, p. 9-20, 2004

MARQUES, M. H. B.; MELO, J. E.; MARTINS, V. A. Madeiras da Amazônia: características e utilização. Brasília: IBAMA, 1997. I4Ip.

MUNSELL, A. H. Munsell book of color: defining, explaining and illustrating the fundamental characteristics of color. Baltimore: Munsell Color Company, 1957.

NASCIMENTO, C. C.; GARCIA, J. N.; DIÁZ, M. P. Agrupamento de espécies madeireiras da Amazônia em função da densidade e propriedades mecânicas. Madera y Bosques, v. 3, n. I, p. 33-52, 1997.
OLIVEIRA, J. T. S. Propriedades físicas e mecânicas da madeira. In: OLIVEIRA, J. T. S.; FIEDLER, N. C.; NOGUEIRA, M. Tecnologias aplicadas ao setor madeireiro. Visconde do Rio Branco: Suprema Gráfica e Editora Ltda, 2007, p. 129-163.

PINHEIRO, A. L.; COUTO, L.; PINHEIRO, D. T.; BRUNETTA, J. M. F. C. Ecologia, silvicultura e tecnologia de utilização dos mognos-africanos (Khaya spp.). Viçosa: Sociedade Brasileira de Agrossilvicultura, 201 I. 102 p.

RINNE, E.; HAKKARAINEN, J.; RIKKINEN, J. Comparative ecological wood anatomy of African mahogany Khaya ivorensis with special reference to damage caused by Hypsipylarobusta. European Journal of Scientific Research, v. 5I, n. I, p. 18-28, 201 I.

THE WOOD DATA BASE. African mahogany. Disponível em: $\quad<$ http://www.wood-database.com/lumberidentification/hardwoods/african-mahogany $>$. Acesso em: 31 out. 2013.

ZBONAK, A.; BROWN, T.; HARDING, K.; INNES, T.; DAVIES, M. Wood properties and processing outcomes for plantation grown African mahogany (Khaya senegalensis) trees from Clare, Queemsland (I 8 and 20-year-old) and Katherine, Northenr Territory (14-year-old). Brisbane: Department of Employment, Economic, Development and Innovation, 2004 